Ross, C. A., Siddievi, A. R. \& MatAs, M. (1987) DSM-III: problems in diagnosis of paranoid and obsessive compulsive disorder. Canadian Journal of Psychiatry, 32, 146-148.

South Shore Hospital and Medical Center

David A. Fishibain

600 Alton Road

Miami Beach

Florida 33139

\section{Tardive dyskinesia, depression and ECT}

SIR: It has been variously reported that ECT may precipitate (Flaherty et al, 1984) or ameliorate (Price \& Levin, 1978) tardive dyskinesia in depressives. Furthermore, Keshavan \& Goswamy (1983) have observed that tardive dyskinesia in bipolar affective illness is worse during the euthymic phase than during the depressive phase. The opposite has also been reported (Cutter et al, 1981).

Case report. An 83-year-old female patient had a six-year history of tardive dyskinesia induced by prochlorperazine maleate prescribed for dizziness at that time. She was admitted with a major depression with psychotic features (DSM-III), (Hamilton Rating Scale for Depression (HRSD; Hamilton, 1960) $=24$ ) and at that time scored 22 on the AIMS scale for tardive dyskinesia. HRSD and AIMS ratings were taken one day before and one day after each ECT treatment and one week after the final ECT (8th unilateral nondominant ECT). AIMS and HRSD scores before and after each of the 3rd to 8th ECT showed a mean fall of 1.4 points on each scale. Overall falls from before ECT to one week after treatment were 24 to 13 for the HRSD, and 22 to 16 for the AIMS.

This patient's tardive dyskinesia was clearly worse during the depressive phase of the illness and improved (but was still present) at recovery. Each treatment with ECT was associated with improvement in mood and tardive dyskinesia and this occurred in parallel. This case study supports the observation that tardive dyskinesia is worse in the depressive phase than in the euthymic phase and that ECT does not worsen the condition. The improvement with ECT may be due to the improvement in the depressive illness but an additional direct effect on tardive dyskinesia may also occur.

Cutter, N. R., Post, R. M., ReY, A. C., et al (1981) Depression dependent dyskinesia in two cases of manic depressive illness. New England Journal of Medicine, 18, 1088-1089.

Flaherty, J. A., NAIDU, J. \& DysBen, M. (1983) Emergent dyskinesia and depression. American Journal of Psychiatry, 141, 808-809.

HAmilton, M. (1960) A rating scale for depression. Journal of Neurology, Neurosurgery and Psychiatry, 23, 56-62.

Keshavan, M. S. \& Goswamy, U. (1983) Tardive dyskinesia less severe in depression. British Journal of Psychiatry, 142, 207.
Price, T. R. P. \& LeVIn, R. (1978) The effects of ECT on tardive dyskinesia. American Journal of Psychiatry, 135, 991-993.

J. A. O. Besson

A. N. Palin

Department of Mental Health

University Medical Buildings

Foresterhill

Aberdeen

A case of depression responding to spontaneous epilepsy but not ECT

SIR: We wish to report the case of a severely depressed man with a long history of manic-depressive psychosis whose depression improved following spontaneous grand-mal epilepsy, but not following repeated electroconvulsive therapy (ECT).

Case report. The patient was a 67 -year-old author, with a 35-year history of manic-depressive psychosis. He had been an in-patient with a resistant psychotic depression for two years before his current episode, but had eventually responded to a combination of amitriptyline, phenelzine, and lithium. He had failed to respond to a course of $10 x$ bilateral ECT given twice weekly. Six months later the patient had a brief manic illness, and then started becoming increasingly depressed again. It was decided to start him on carbamazepine, initially $100 \mathrm{mg}$ t.d.s. and then $200 \mathrm{mg}$ t.d.s. Three weeks after starting carbamazepine his condition deteriorated further, and he had a depressive psychosis with profound psychomotor retardation and nihilistic delusions. He stopped eating and taking medication. ECT was instituted, but the first ECT failed to produce any improvement. Some three nights later the patient had a total of five spontaneous grand-mal seizures lasting 1-2 minutes before responding to intravenous valium. Despite full investigation, no cause for these was found and they were ascribed to acute carbamazepine withdrawal, which was not restarted.

Some 36 hours following the spontaneous epileptic seizures the patient's mood was noted to be radically different. He was no longer depressed, was laughing and talking appropriately, and was eating and drinking normally. Over the following 10 days his condition deteriorated and it was decided to reinstitute ECT. Over the next six weeks the patient had a further 11 ECTs, which all produced bilateral seizures lasting at least 25 seconds, but he did not improve. The patient's depression again proved resistant, only improving some eight months later on high doses of tricyclic antidepressants and monoamine oxidase inhibitors.

As far as we are aware, this is the first reported case in which spontaneous grand-mal seizures have improved the clinical state of a patient with depression, while ECT has had no effect. This differential response may possibly have been due to either the differences in time course between the spontaneous and induced seizures, or because the patient received 